\title{
SUFFICIENT CONDITIONS FOR SPIRAL-LIKENESS
}

\section{H. SILVERMAN}

Department of Mathematics

College of Charleston

Charleston, SC 29424

(Received December 13, 1988)

ABSTRACT. Coefficient conditions sufficient for spiral-likenss are found by convolution methods. The order of starlikeness for such functions is also determined.

KEY WORDS AND PHRASES. Spiral-11ke, starlike, convolution.

1980 AMS SUBJECT CLASSIFICATION CODES. 30C45, $30 \mathrm{C5} 5$.

1. INTRODUCTION.

A function $f(z)=z+\sum_{n=2}^{\infty} a_{n} z^{n}$ analytic in the unit disk $\Delta=\{|z|<1\}$ is said to be starlike of order $\alpha, 0<\alpha<1$, if $\operatorname{Re}\left\{\mathrm{zf}^{\prime} / \mathrm{f}\right\}>\alpha, z \varepsilon \Delta$, and is said to be $\lambda$ spiral-1ike, $-\pi / 2<\lambda<\pi / 2$, if $\operatorname{Re}\left\{e^{i \lambda} f^{\prime} / f\right\}>0, z \varepsilon \Delta . \quad$ We denote these classes, respectively, by $S^{*}(\alpha)$ and $S p(\lambda)$. Note that $S p(0)=S^{*}(0)$, the family of starlike functions. Functions in $S p(\lambda)$ were shown by Spacek [4] to be univalent in $\Delta$ and were later studied extensively by Libera [1].

A function $f$ is in $S *(\alpha)$ if its coefficients are sufficiently small.

THEOREM A [2] . If the coefficients of $f(z)=z+\sum_{n=2}^{\infty} a_{n} z^{n}$ satisfy the inequality

$$
\sum_{n=2}^{\infty} \frac{n-\alpha}{1-\alpha}\left|a_{n}\right|<1,
$$

then $\left|\left(z f^{\prime} / f\right)-1\right|<1-\alpha, z \varepsilon \Delta$, and hence $f \varepsilon S^{*}(\alpha)$.

It is our purpose here to find coefficient conditions guaranteeing that $f$ is in

$\mathrm{Sp}(\lambda)$. Our methods will involve convolution properties and will also furnish us with an alternate proof that (1) is a sufficient condition for $f$ to be in $S^{*}(\alpha)$.

The convolution or Hadamard product of two power series $f(z)=\sum_{n=0}^{\infty} a_{n} z^{n}$

and $g(z)=\sum_{n=0}^{\infty} b_{n} z^{n}$ is defined as the power series ( $\left.f * g\right)(z)=\sum_{n=0}^{\infty} a_{n} b_{n} z^{n}$. In [3] it is shown that $f \in S *(\alpha)$ if and only if

and $f \in S p(\lambda)$ if and only if

$$
\frac{1}{z}\left(f * \frac{z+((x+2 \alpha-1) /(2-2 \alpha)) z^{2}}{(1-z)^{2}}\right) \neq 0 \quad(z \varepsilon \Delta,|x|=1)
$$

$$
\frac{1}{z}\left(f * \frac{z+\left(\left(x-e^{-21 \lambda}\right) /\left(1+e^{-21 \lambda}\right)\right) z^{2}}{(1-z)^{2}}\right) \neq 0 \quad(z \varepsilon \Delta,|x|=1) \text {. }
$$

Now $\left(z+c z^{2}\right) /(1-z)^{2}=z+\sum_{n=2}^{\infty}(n+(n-1) c) z^{n}$, so we may restate these results as 
THEOREM B. The function $f(z)=2+\sum_{n=2}^{\infty} a_{n} z^{n} \in S^{*}(\alpha)(S p(\lambda))$ if and only if $1+\sum_{n=2}^{\infty}(n+(n-1) c) a_{n} z^{n-1} \neq 0$ for al1 $\geq \varepsilon \Delta$ and $|x|=1$, where

$$
c=\frac{x+2 \alpha-1}{2-2 \alpha}\left(c=\frac{x-e^{-21 \lambda}}{1+e^{-21 \lambda}}\right)
$$

Since $\left|1+{ }_{n=2}^{\infty}(n+(n-1) c) a_{n} z^{n-1}\right|>1-\sum_{n=2}^{\infty}|n+(n-1) c|\left|a_{n}\right||z|^{n-1}$,

a sufficient condition for $f$ to be $\ln S^{*}(\alpha)$ or $\operatorname{Sp}(\lambda)$ is that $\sum_{n=2}^{\infty}|n+(n-1) c|\left|a_{n}\right|<1$ for the appropriate choice of c. A straightforward computation shows that

$$
\left|n+\frac{(n-1)(x+2 \alpha-1)}{2-2 \alpha}\right|<\left|n+\frac{(n-1)(2 \alpha)}{2-2 \alpha}\right|=\frac{n-\alpha}{1-\alpha},
$$

and we can conclude from Theorem $B$ that condition (1) is sufficient for $f$ to be in $S^{\star}(\alpha)$. The corresponding result for $\operatorname{Sp}(\lambda)$ is computationally more involved.

2. THE MAIN CLASS.

THEOREM 1. The function $f(z)=z+\sum_{n=2}^{\infty} a_{n} z^{n} \in S p(\lambda) \underline{\text { if }} \sum_{n=2}^{\infty} B_{n}(\lambda)\left|a_{n}\right|<1$ for

$$
B_{n}(\lambda)=\frac{(n-1)+\sqrt{(n-1)^{2}+4 n \cos ^{2} \lambda}}{2 \cos \lambda}
$$

The result 18 sharp, with $f_{n}(z)=z+a_{n} z^{n}$ In $\operatorname{Sp}(\lambda)$ 1f and only if $\left|a_{n}\right|<1 / B_{n}(\lambda)$.

PROOF. From Theorem B, it suffices to show for $c=\left(x-e^{-21 \lambda}\right) /\left(1+e^{-21 \lambda}\right)$ that

$$
|x|=1 \text { max }|n+(n-1) c|=B_{n}(\lambda) \text {. Writing } c=c_{1}+1 c_{2}, \quad c_{1} \text { and } c_{2} \text { real, we have }
$$

$$
|n+(n-1) c|=\sqrt{n^{2}+(n-1)\left[(n-1)\left(c_{1}^{2}+c_{2}^{2}\right)+2 n c_{1}\right] . ~}
$$
Hence $|n+(n-1) c|$ will attain 1 ts maximum when $(n-1)\left(c_{1}^{2}+c_{2}^{2}\right)+2 n c_{1}$ does. Setting
$x=e^{1 \beta}$, a computation shows that

$$
c_{1}^{2}+c_{2}^{2}=\frac{1-\cos (2 \lambda+\beta)}{1+\cos 2 \lambda} \text { and } c_{1}=\frac{\cos (2 \lambda+\beta)+\cos \beta-(1+\cos 2 \lambda)}{2(1+\cos 2 \lambda)} \text {. }
$$

Thus

$$
(n-1)\left(c_{1}^{2}+c_{2}^{2}\right)+2 n c_{1}=\frac{\cos (2 \lambda+\beta)+n \cos \beta(1+n \cos 2 \lambda)}{2(1+\cos 2 \lambda)},
$$

which is maximized when $g(\beta)=\cos (2 \lambda+\beta)+n \cos \beta$ is maximized. But $g(\beta)$ attains its maximum when $\beta=\beta_{0}=\tan ^{-1}\left(-\frac{\sin 2 \lambda}{n+\cos 2 \lambda}\right)$, with $g\left(\beta_{0}\right)=\sqrt{(n-1)^{2}+4 n \cos ^{2} \lambda}$. For this choice of $\beta_{0}$, we have

$$
(n-1)\left(c_{1}^{2}+c_{2}^{2}\right)+2 n c_{1}=\frac{\sqrt{(n-1)^{2}+4 n \cos ^{2} \lambda}+(n-1)-2 n \cos ^{2} \lambda}{2 \cos ^{2} \lambda}=t_{n}(\lambda) \text {. }
$$


It now follows from (2) that

$$
\begin{aligned}
& |x|=1|n+(n-1) c|=\left(n^{2}+(n-1) t_{n}(\lambda)\right)^{1 / 2}, \\
& =\left(\frac{(n-1)^{2}+2 n \cos ^{2} \lambda+(n-1) r(n-1)^{2}+4 n \cos ^{2} \lambda}{2 \cos ^{2} \lambda}\right)^{1 / 2} .
\end{aligned}
$$

which may be expressed as $B_{n}(\lambda)$. This completes the proof. To show sharpness, note that according to Theorem $B f_{n}(z)=z+a_{n} z^{n} \notin \operatorname{Sp}(\lambda)$ if $z^{n-1}=-\left[(n+(n-1) c) a_{n}\right]^{-1}$ has a solution for $z \in \Delta$. Choosing $c$ so that $|n+(n-1) c|=B_{n}(\lambda)$, we see that $f_{n}(z) \notin S p(\lambda)$ if $\left|a_{n}\right|>1 / B_{n}(\lambda)$. In particular, $f_{n}(z)=z+a_{n} z^{n} \varepsilon S p(\lambda)$ if and on $1 y$ if $\left|a_{n}\right|<1 / B_{n}(\lambda)$.

COROLLARY 1. If $f_{n}(z)=z+a_{n} z^{n} \varepsilon \operatorname{Sp}\left(\lambda_{0}\right)$, then $f_{n} \varepsilon \operatorname{Sp}(\lambda)$ for $|\lambda|<\left|\lambda_{0}\right|$. PROOF. This is a consequence of $B_{n}(\lambda)$ being an increasing function of $|\lambda|$.

In fact, any function that satisfles the conditions of Theorem 1 for $\lambda=\lambda_{0}$ will also be in $\mathrm{Sp}(\lambda)$ for $|\lambda|<\left|\lambda_{0}\right|$, a sharp contrast to the inclusion properties for the general class $\operatorname{Sp}(\lambda)$. The function

$f_{\lambda}(z)=z(1-z)^{-2 e^{-1 \lambda}} \cos \lambda$ is in $\operatorname{Sp}(\lambda)$ but it is not in $\operatorname{Sp}(\lambda)$ for any $\gamma \neq \lambda$. On the other hand, the upper bound on the modulus of the coefficients for $f \varepsilon \operatorname{Sp}(\lambda)$ is a decreasing function of $|\lambda|$. Zamorski [5] showed the sharp coefficient bounds $\left|a_{n}\right|$ for f $\varepsilon \operatorname{Sp}(\lambda)$ to be

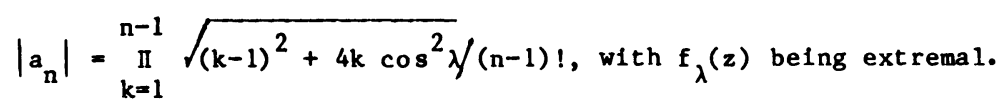

Though Theorem 1 gives a sharp result, it is not aesthetically pleasing because of the complicated nature of $B_{n}(\lambda)$. A consequence of the inequality $1+(n-1) \sec \lambda>B_{n}(\lambda)$ is more palatable sufficient condition.

COROLLARY 2. If $\sum_{n=2}^{\infty}(1+(n-1) \sec \lambda)\left|a_{n}\right|<1$, then $f(z)=z+{ }_{n=2}^{\infty} a_{n} z^{n} \varepsilon \operatorname{sp}(\lambda)$. Using a different method, Corollary 2 will also be shown to follow from Theorem 3 . 3. ORDER OF STARLIKENESS.

Since $B_{n}(\lambda)>n$, we see from Theorem $A$ that a function satisfying the conditions of Theorem 1 must be starlike. We can actually do better.

THEOREM 2. If $f$ satisfies the conditions of Theorem 1 then $f \varepsilon S^{*}\left(\alpha_{0}\right)$ for

$$
\begin{aligned}
& \alpha_{0}=\left(3-\sqrt{\left.1+8 \cos ^{2} \lambda\right) / 2(1+\cos \lambda) \text {. The result is sharp, with extremal function }}\right. \\
& f_{2}(z)=z+z^{2} / B_{2}(\lambda) .
\end{aligned}
$$

PROOF. In view of Theorem $A$, we need only show that $B_{n}(\lambda)>\left(n-\alpha_{0}\right) /\left(1-\alpha_{0}\right)$ for every $n$. Since $B_{2}(\lambda)=\left(2-\alpha_{0}\right) /\left(1-\alpha_{0}\right)$, it suffices to prove that $\left(1-\alpha_{0}\right) B_{n}(\lambda) /\left(n-\alpha_{0}\right)$ is an increasing function of $n$. Setting

$$
G(x)=\frac{x-1+\sqrt{(x-1)^{2}+4 x \cos ^{2} \lambda}}{x-\alpha_{0}} \text {, I will show that } G^{\prime}(x)>0 \text { for } x>2 \text {. }
$$


d differentiation of $G$ leads to

$=\mathrm{H}(\mathrm{x})$, say.

$$
\left(x-\alpha_{0}\right)^{2} G^{\prime}(x)=1-\alpha_{0}+\left[\frac{\left(1-\alpha_{0}-2 \cos ^{2} \lambda\right) x-\left(1-\alpha_{0}+2 \alpha_{0} \cos ^{2} \lambda\right)}{\sqrt{(x-1)^{2}+4 x \cos ^{2} \lambda}}\right]
$$

Since $H^{\prime}(x)=\frac{4 \sin ^{2} \lambda \cos ^{2} \lambda\left(x-\alpha_{0}\right)}{\left((x-1)^{2}+4 x \cos ^{2} \lambda\right)^{3 / 2}}>0$, it follows for $x>2$ that

$$
H(x)>H(2)=\cos \lambda\left(1-\frac{4 \cos \lambda-1}{\sqrt{1+8 \cos ^{2} \lambda}}\right)>0 \text {. }
$$

Therefore $G(x)$, and consequently $\left(1-\alpha_{0}\right) B_{n}(\lambda) /\left(n-\alpha_{0}\right)$, is an increasing function. This completes the proof.

We have actually shown more according to Theorem A.

COROLLARY. If $f$ satisfies the conditions of Theorem 1 , then $\left|\left(z f^{\prime} / f\right)-1\right|<1-\alpha_{0}, z \varepsilon \Delta$.

Functions in $S^{\star}(\alpha)$ need not be $\ln \operatorname{Sp}(\lambda)$ for $\lambda \neq 0$. The function $f_{\alpha}(z)=z /(1-z)^{2(1-\alpha)} \varepsilon S^{\star}(\alpha)$ since $z f_{\alpha}^{\prime} / f_{\alpha}$ maps $\Delta$ onto the half plane Re w> $\alpha$. However $f_{\alpha} \nmid S_{p}(\lambda), \lambda \neq 0$. We next look at a subclass of $s^{\star}(\alpha)$ whose functions are spiral-1ike.

THEOREM 3. If $f(z)=z+\ldots$ is analytic with $\left|\left(z f^{\prime} / f\right)-1\right|<1-\alpha$ for $z \varepsilon \Delta$, then $f \in \operatorname{Sp}(\lambda)$ for $|\lambda|<\cos ^{-1}(1-\alpha)$. The result is sharp, with extremal function $f(z)=z e^{(1-\alpha) z}$.

PROOF. We may write $\left(z f^{\prime} / f\right)-1=(1-\alpha) \omega(z)$, where $|\omega(z)|<1$ for $z \varepsilon \Delta$. Thus $\operatorname{Re}\left\{e^{i \lambda} z f^{\prime} / f\right\}=\cos \lambda+(1-\alpha) \operatorname{Re}\left\{e^{i \lambda} \omega(z)\right\}>\cos \lambda-(1-\alpha)\left|e^{1 \lambda} \omega(z)\right|>$ $\cos \lambda-(1-\alpha)>0$ for $|\lambda|<\cos ^{-1}(1-\alpha)$, and the proof is complete.

COROLLARY. If $\left|\left(z f^{\prime} / f\right)-1\right|<\cos \lambda$, then $f \varepsilon \operatorname{Sp}(\lambda)$.

PROOF. Set $\alpha=1-\cos \lambda$ in Theorem 3 .

Finally an application of Theorem A, with $\alpha=1-\cos \lambda$, to Theorem 3 provides us with an alternate proof to Corollary 2 of Theorem 1.

\section{REFERENCES}

1. LIBERA, R.J., Univalent a-spiral functions, Canad. J. Math. 19 (1967), 449-456.

2. SILVERMAN, H., Univalent functions with negative coefficients, Proc. Amer. Math. Soc. 51 (1975), 109-116.

3. SILVERMAN, H., SilviA, E.M. and TELAGE, D.N., Convolution conditions for convexity, starlikeness, and spiral-1ikeness, Math Zeitschrift 162 (1978), 125-130.

4. SPAČK, L., Přrispěvek k teoril funcki prostyč, căsopis Pěst Mat. a Fys.62 (1932), 12-19.

5. ZAMORSKI, J., About the extremal spiral schlicht functions, Ann. Polon, Math.9 (1962), 265-273. 


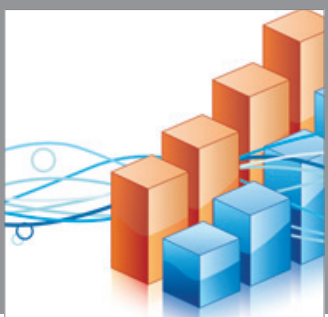

Advances in

Operations Research

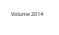

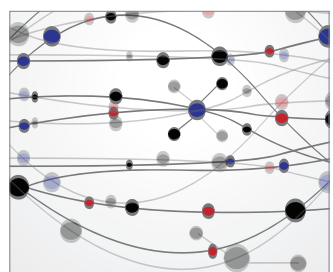

\section{The Scientific} World Journal
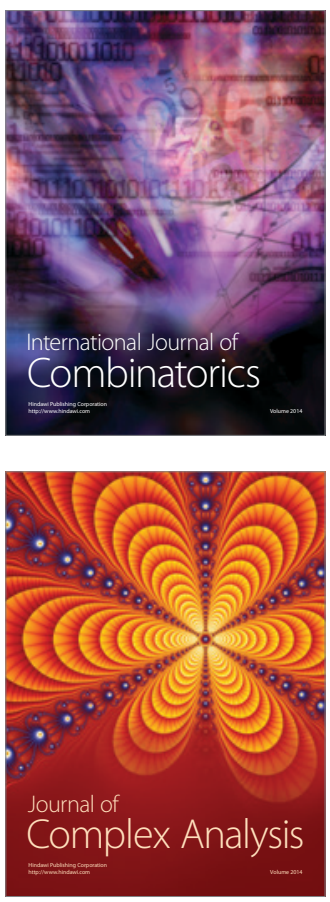

International Journal of

Mathematics and

Mathematical

Sciences
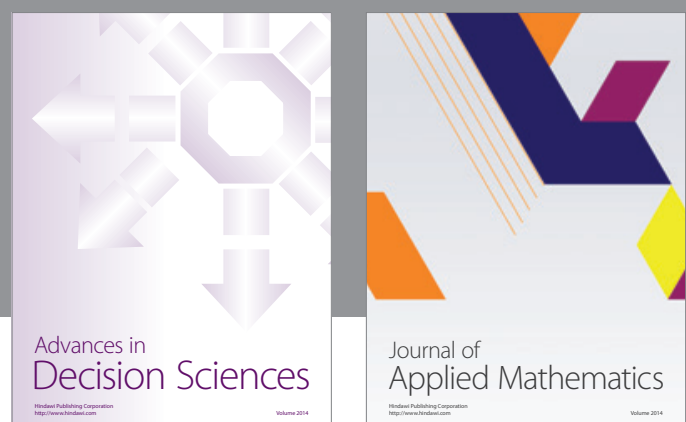

Journal of

Applied Mathematics
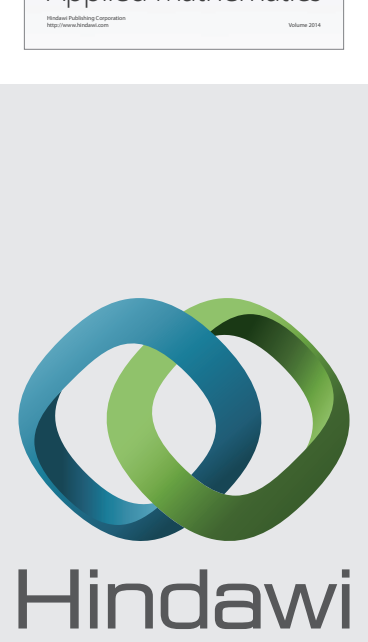

Submit your manuscripts at http://www.hindawi.com
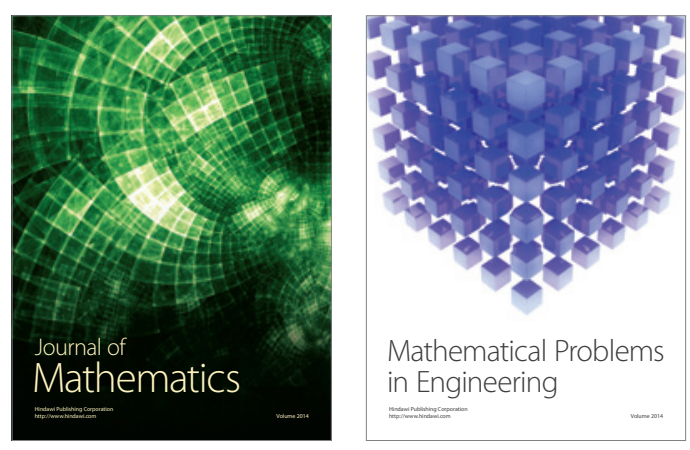

Mathematical Problems in Engineering
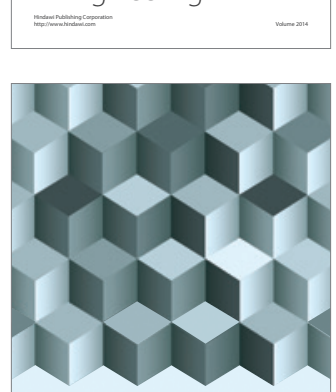

Journal of

Function Spaces
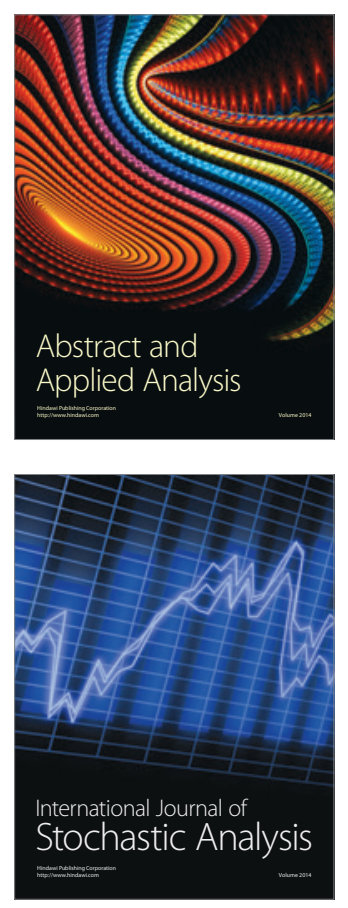

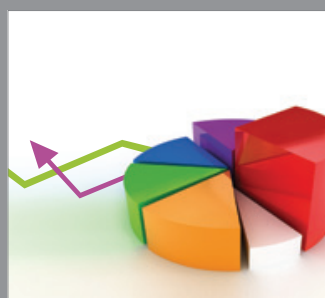

ournal of

Probability and Statistics

Promensencen
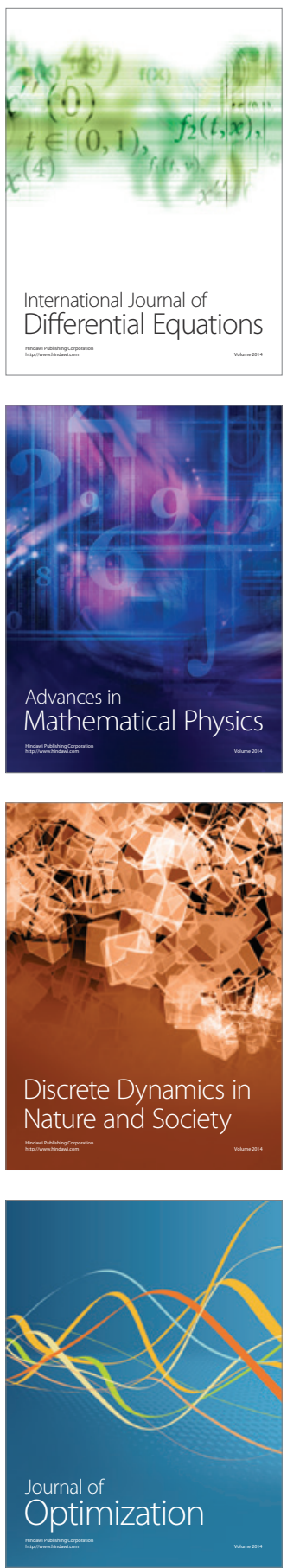